\section{The use of the Index of Orthodontic Treatment Need in dental primary care}

\author{
J. Ho-A-Yun, ${ }^{1}$ F. Crawford ${ }^{2}$ and J. Clarkson ${ }^{3}$
}

IN BRIEF

- Examines the extent to which the IOTN is used in general and specialist orthodontic dental practice in Scotland.

- Identifies variations in the clinical practice of these two groups of dental practitioners.

- Considers the barriers which might impede the implementation process. Makes recommendations for the implementation of the IOTN into clinical practice.

\begin{abstract}
Objectives The primary objective of this survey was to assess the use of the Index of Orthodontic Treatment Need (IOTN) in dental primary care (Scottish general dental services - SGDS), and to compare orthodontic specialists (OS) and general dental practitioners (GDPs). The secondary objective was to explore the attitudes to mandatory introduction of the IOTN into the SGDS. Design Postal, self completed questionnaire. Setting Dental primary care, Scotland. Subjects Randomly selected sample of general dental practitioners (GDPs), $n=315$, and all orthodontic specialist practitioners (OS), $n=49$, identified as working in the SGDS. Main outcome measures Prevalence and experience of using the IOTN in the SGDS. Results Response rate was $46 \%(n=169)$. Eighty-four percent of respondents did not use the IOTN. Thirty-five percent of respondent GDPs had never heard of the IOTN. Respondents reported using the IOTN as an inter-colleague communication tool and to grade case complexity. GDPs perceived the IOTN as beneficial in setting national standards of practice; OS saw it as a tool to justify the allocation of NHS resources to patients. Responses indicate concerns that IOTN introduction will restrict access to orthodontic care. Conclusions The IOTN is not widely used in the SGDS but is perceived as standardising treatment need assessment by GDPs and justifying the allocation of NHS orthodontic resources to patients amongst OS. Introduction of mandatory IOTN grading is likely to be contentious - the following needs to be considered: the index profile should be raised; its advantages highlighted; concerns about restricted access to orthodontic care addressed; and perceived need for locally accessed training met.
\end{abstract}

\section{BACKGROUND}

Malocclusions present as a spectrum of dental traits rather than a diseasespecific condition. Where finite public resources exist, assessment of these must support cost effective treatment decisions. ${ }^{1}$ Ideally such assessments should be objective, emphasising the need to use a health measurement scale or index.

The use of an index should achieve the following objectives: greater professional uniformity, standardisation in assessing orthodontic treatment need,

$1^{*}$ Orthodontic Department, Victoria Hospital, Hayfield Road, Kirkcaldy, KY2 5AH; ${ }^{2}$ Senior Research Fellow/ Honorary Senior Lecturer, General Practice Section, The University of Edinburgh, 20 West Richmond Street, Edinburgh, EH8 9DX; ${ }^{3}$ Senior Lecturer/Consultant, The Dental Health Services Research Unit, The University of Dundee, The Mackenzie Building, Kirsty Semple Way, Dundee, DD2 4BF

*Correspondence to: Mr John Ho-A-Yun Email: John.Hoayun@faht.scot.nhs.uk

\section{Online article number E16}

Refereed Paper - accepted 22 September 2008

DOI: $10.1038 /$ sj.bdj.2009.310

${ }^{\circledR}$ British Dental Journal 2009; 206: E16 selective resource allocation, reduced risk of unnecessary orthodontic treatment, ${ }^{2}$ and equity of access. Many indices exist but none are recognised as the international 'gold standard'.'

In the UK the Index of Orthodontic Treatment Need (IOTN) was developed to provide an objective measure of orthodontic treatment need. Malocclusion severity is graded on two component scales: firstly dental health (DHC), grading 1 to 5. A letter notation is added to indicate malocclusion trait. Secondly, an aesthetic (AC) grading from 1 to 10 can be scored. ${ }^{3}$ These grades can be grouped thus: little/no need - DHC 1, 2 (AC 1-4); possible/moderate need - DHC 3 (AC 57); and great/definite need - DHC 4 and 5 (AC 7-10). ${ }^{4}$ By using these sub-groupings to set a treatment cut-off grade or threshold, patients can be prioritised for the allocation of finite NHS resources. ${ }^{5}$

IOTN use was estimated to be $33 \%$ in 'main UK secondary care centres' in $1991 .^{6}$ This increased to an estimated $75 \%$ by $1994 .^{7}$ In 2003 a survey of UK public dental health consultants reported that the IOTN was used by $80 \%$ of respondents. Usage reportedly centres on waiting list control in secondary care, particularly where local health authorities have set treatment threshold grades. ${ }^{7}$ Public health consultants use the IOTN for planning, contracting and monitoring orthodontic services. ${ }^{2}$

A pilot for primary care, based on a personal dental service contract, used the IOTN to allocate resources. There were two key findings: treatment waiting times were reduced, and the IOTN proved to be suitable for use in a general dental services (GDS) setting. ${ }^{8}$

In Scotland it is estimated that $90 \%$ of dental care takes place in general dental practices. The remaining $10 \%$ of care takes place in specialist practices and the secondary care sector. ${ }^{9}$ These specialist services are mainly in the field of orthodontics. ${ }^{10}$ The Scottish Executive highlights the need for 'value for money in the use of public resources', ie resource allocation based on treatment need. ${ }^{9}$ In 
Norway and Sweden, orthodontic indices are employed for this purpose. ${ }^{11,12}$

Currently, dentists are not obliged to use the IOTN in the Scottish general dental services (SGDS). Anecdotal evidence suggested that few if any practitioners use the IOTN.

The primary aim of this survey was to assess and compare the reported use of the IOTN by general dental practitioners (GDPs) and orthodontic specialists (OS) in the SGDS. Secondary aims were to highlight reasons behind use and non-use. Lastly, it aimed to explore attitudes to the potential introduction of the index as a mandatory part of SGDS orthodontic provision.

\section{METHODS}

The methods, conduct and design of the survey were informed by systematic reviews, ${ }^{13,14}$ a narrative review ${ }^{15}$ and health measurement scale development. ${ }^{16}$

We undertook a postal questionnaire survey of a randomly selected sample of GDPs and all of the OS identified as working in the SGDS. The extent to which the IOTN is used by these practitioners was assessed, and practitioners' views about this health measurement scale were elicited. To inform the survey, two pilot studies were conducted via the local primary care research network (East ReN, formerly Tay ReN).

\section{Pilot study 1}

The first pilot sourced items for the questionnaire - questions and responses. In order for these to be relevant and valid, a purposive sample was drawn from potential respondents to the main survey. ${ }^{16}$ GDPs ( $n=5)$ were contacted through the local research network (East ReN).

An initial topic guide was developed $^{17}$ prior to conducting face-to-face semi-structured interviews. No assumptions were developed a priori in line with grounded theory. Interviews were reflexive in nature. ${ }^{18}$ All themes, however 'extreme', were included for analysis in accordance with fair dealing. ${ }^{18,19}$

Salient points from the topic guide were entered into each subsequent guide prior to the next interview. Inductive analysis was carried out on the collated notes using a four step process: ${ }^{20}$ immersion, deriving emergent themes, coding,

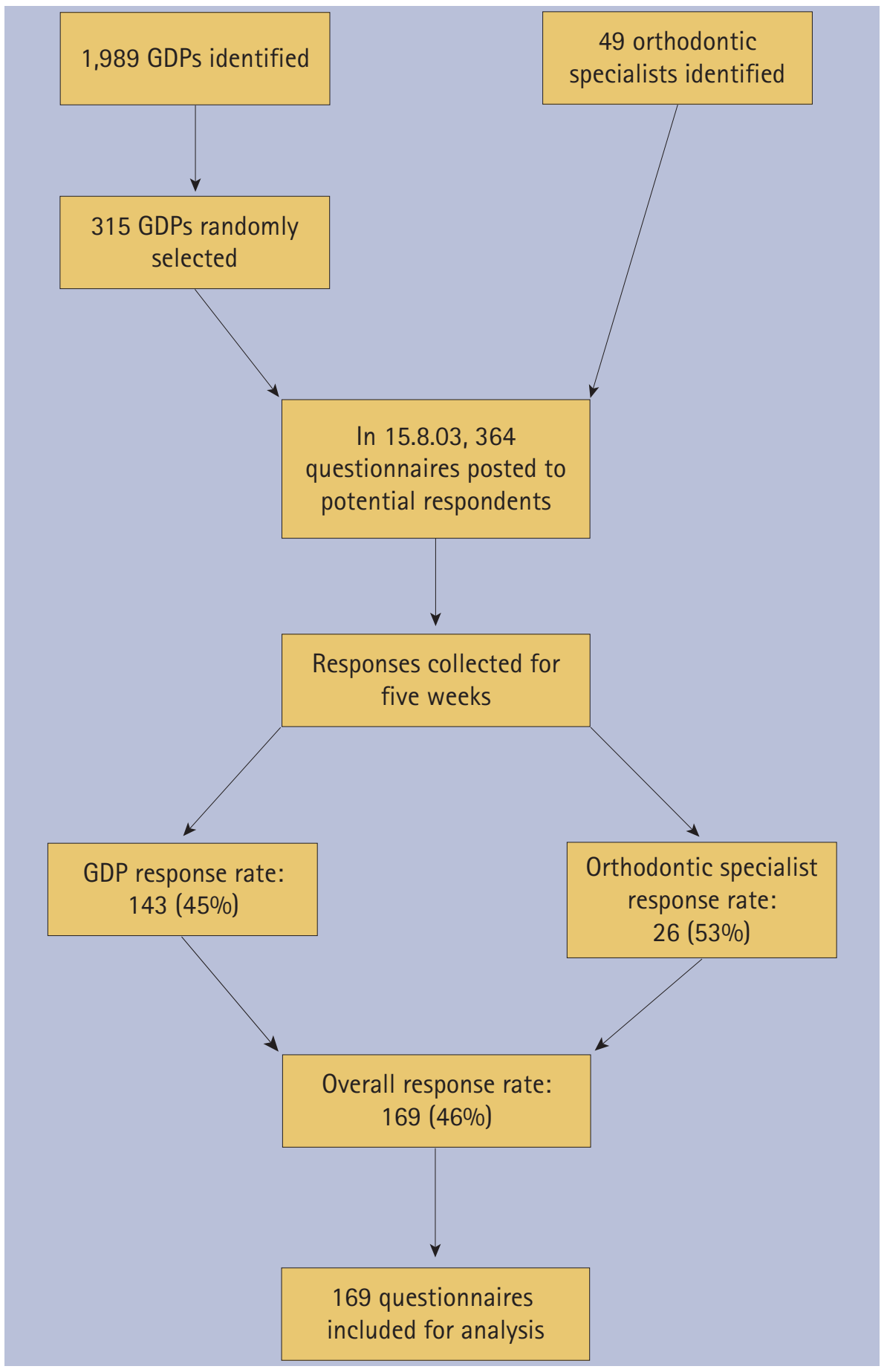

Fig. 1 Flow chart of participants in survey

and finally synthesis into a questionnaire. The pilot questionnaire consisted of ten closed response format questions. It included response items generated by pilot participants and researchers.

\section{Pilot study 2}

A purposive sample of GDPs was sent questionnaires and covering letters via Tay ReN ( $\mathrm{n}=30)$. Response rate was $47 \%(n=14)$. No problems with content were noted. Cronbach's alpha was used to test reliability (score of 0.46 ). It was decided at this stage to adopt an anonymous response strategy for the final questionnaire.

\section{Questionnaire content}

The final questionnaire contained four sections: 1. IOTN use: current, previous/ ceased or not at all; 2. How respondents used IOTN; 3. Attitudes towards mandatory introduction of IOTN; 4. Demographic information. The content of these sections was screened into three 'question pathways' based on section 1 responses. 


\begin{tabular}{|c|c|c|}
\hline Item & $\begin{array}{l}\text { GDPs } \\
n=143\end{array}$ & $\begin{array}{l}\text { OS } \\
n=26\end{array}$ \\
\hline Current use & 14 (10\%) & $13(50 \%)$ \\
\hline Ceased use & $25(17 \%)$ & $10(38 \%)$ \\
\hline Never used & 104 (73\%) & $3(12 \%)$ \\
\hline
\end{tabular}

\begin{tabular}{|c|c|c|}
\hline Item & $\begin{array}{l}\text { GDPs } \\
\mathrm{n}=104\end{array}$ & $\begin{array}{l}\text { OS } \\
n=3\end{array}$ \\
\hline Not heard of IOTN & $50(48 \%)$ & 0 \\
\hline Lack of training & $47(45 \%)$ & $2(66 \%)$ \\
\hline Lack of specific fee & $23(22 \%)$ & $1(33 \%)$ \\
\hline Not mandatory & 20 (19\%) & $1(33 \%)$ \\
\hline Other priorities & $15(14 \%)$ & $1(33 \%)$ \\
\hline Too time consuming & $12(12 \%)$ & 0 \\
\hline Unsuitable for NHS use & $9(9 \%)$ & 0 \\
\hline Too complex & $3(3 \%)$ & 0 \\
\hline Other & $12(12 \%)$ & 0 \\
\hline
\end{tabular}

\section{The sampling frame}

The sampling frame was the Scottish general dental practitioners (SGDP) register (source: Dental Practitioner Board ISD). All practitioners (GDPs and orthodontic specialist practitioners - OS) with a current Scottish health board number were considered eligible to receive the questionnaire ( $\mathrm{n}=1,989)$. This was cross-referenced with the GDC specialist register to identify 49 OS. All OS were included in the study.

\section{Ethical approval}

The local ethics committee and MREC advised no ethical approval was required.

\section{Survey outcome measures}

The primary outcome measured was use of the IOTN in Scotland. Secondary outcomes measured were the attitudes of respondents to the index.

\section{Sample size}

An a priori sample size calculation of 315 based on $8 \%$ use of IOTN, with a standard deviation of 0.03 , gave $80 \%$ power to detect a 10\% difference between GDPs and OS. Computer software (Excel 2000) was used to randomise the SGDP list order and then randomly select the 315 potential respondents. An independent researcher carried this out.

\section{Statistical methods}

Data from the questionnaires were coded and entered into SPSS version 11 for analysis. Cross-tabs were created and $\chi^{2}$ was used to test for statistically significant differences at the 95\% level. Continuity correction and Fisher's exact test were used where appropriate.

\section{RESULTS}

Three hundred and sixty-four questionnaires were posted for self-completion (Fig. 1). Responses to the questions are shown below. $P$ values are given where a statistical difference between GDPs and OS was detected at $\mathrm{p}<0.05$ (Tables 1 and 2).

\section{Response rates}

Overall response rate was 46\% ( $\mathrm{n}=$ 169). The rate for GDPs was $45 \%$ ( $\mathrm{n}=$ 143) and for orthodontic specialists 53\% $(\mathrm{n}=26)(\mathrm{p}<0.001)$. All responses were anonymous.

The majority of respondents were male (74\%). Median range for duration of practice was between 14 and 23 years; mean number of years since qualification was 20. They were most likely to have qualified from one of three Scottish universities: Glasgow (47\%), Edinburgh (24\%), and Dundee (22\%). They worked mainly in NHS (78\%) and urban settings (65\%).

\section{IOTN use}

Sixteen percent of all respondents currently use the IOTN. This figure consists of 50\% OS ( $\mathrm{n}=13$ ) and 10\% GDPs ( $\mathrm{n}=$ 14). Denominators are given at the end of each paragraph in italics: GDPs $n=$ 143, OS $n=26$. (Table 1).

Reasons given for currently using the IOTN were reportedly for communication with colleagues and patients, and 64\% of respondent GDPs used it for grading case complexity. GDPs $n=14$, OS $n=13$.

Respondents gave various reasons for stopping using the IOTN. GDPs stopped using the IOTN mainly because there is no fee. The OS have ceased use because it is not mandatory. Only GDPs gave any free text responses to this question: the IOTN was used solely during undergraduate training. Potential orthodontic cases were referred to an OS for assessment, and therefore the GDP had ceased to use IOTN. GDPs $n=25$, OS $n=10$.

Forty-eight percent of GDPs had never heard of the IOTN and had therefore never used it. Lack of the relevant training was the other main reason for never using the IOTN.

Free text responses elicited the following: again, cases were referred by GDPs to OS for assessment. One respondent cited the lack of relevant AC photographs. GDPs $n=104$, OS $n=3$ (Table 2).

OS were more likely to have used the index previously than GDPs, 88\% versus $27 \%$ respectively. This was statistically significant, $\mathrm{p}<0.001$.

\section{Mandatory introduction}

Setting national practice standards was perceived as most beneficial by $61 \%$ of respondent GDPs, but only 35\% of OS $(p=0.009)$. OS thought the IOTN would be a useful tool in communicating to patients how NHS resources were allocated. GDPs $n=143$, OS $n=26$.

Almost equal proportions from each respondent group thought the IOTN introduction would bring about restricted access to NHS orthodontic care. There 
was concern from GDPs and OS that the IOTN could reduce clinical autonomy in decision-making. GDPs $n=143$, OS $n=$ 26.

When asked: 'Should health authorities set a treatment cut-off grade, with only those patients allocated this grade or higher, receiving NHS orthodontic treatment?', the majority of respondents (51\%) did not believe that health authorities should set a treatment cut-off grade. GDPs $n=143$, OS $n=26$.

Both respondent groups expressed a preference for IOTN courses which could be accessed locally. GDPs favoured journal articles as a source of IOTN education. Twenty-seven percent of GDPs and $35 \%$ of OS thought that existing courses needed better publicity. GDPs $n=143$, OS $n=26$.

\section{DISCUSSION}

Surveys with low response rates have low precision; response rates of $>70 \%$ are desirable. However, the majority of postal surveys published in the medical literature do not achieve this level. ${ }^{13}$

The response rate of this survey was $46 \%$. Questionnaires which are perceived as irrelevant or unimportant yield a lower response rate than those judged salient. ${ }^{21}$ This would appear to be true of the IOTN: it demonstrably lacks relevance to the clinical practice of the GDPs surveyed. A supporting indicator is the statistically significant difference detected in the response rates between GDPs and orthodontic specialists (8\% difference, $\mathrm{p}<0.001$ ).

Strategies to maximise response size were inherent in the design of the survey questionnaire: anonymity may be a motivational factor in survey research to increase response. ${ }^{13}$ This survey was conducted anonymously, therefore was it not possible to link a questionnaire with a specific respondent. Reminders could not be targeted, nor was it possible to identify systematic differences between the respondents and nonrespondents. Blanket repeat mailing of all the respondents carries the risk of repeat responders, which would confound the data. Sensitivity analysis is only possible where variables from the non-respondents have been collected.

Survey research into response rates of health professionals has shown that lower response rates have been observed amongst older doctors and those with fewer qualifications. ${ }^{13}$

A single mailing was utilised in this survey to generate baseline data for a related publication. This demonstrated that a statistically significant improvement in response rates can be achieved in a survey conducted in dental practice, using a telephone prompt. ${ }^{22}$ Lack of baseline data, when considering response enhancing strategies, has been highlighted; single mailing methods are used to generate this. ${ }^{14}$

The Cronbach's alpha score represents a lower score than is conventional, however lower statistical reliability is deemed acceptable where further refinements might sacrifice the breadth of topics covered, ie high content validity. ${ }^{16}$ The three 'question pathways' may also have affected the overall score as each investigated discrete themes surrounding the IOTN. When calculated individually the pathways achieved scores of between 0.6 and 0.7, indicating higher statistical reliabilities.

The IOTN has achieved poor penetration into the SGDS setting, particularly among GDPs. A statistically higher percentage of OS had used the index previously. One explanation for this is that OS encountered the IOTN in their postgraduate specialist training. Two main questions were addressed in the survey: firstly, what is the index used for, and secondly, why do the majority of the respondents not use it?

The IOTN was used for varied purposes. The most prevalent use was for inter-colleague communication. Also the index was, inappropriately, used to grade case complexity. The index's inability to grade potential complexity has been highlighted as a weakness. ${ }^{2,3}$ The reported use in this survey is inconsistent with evidence from surveys conducted in secondary ${ }^{7}$ and tertiary care. ${ }^{2}$

Over a third of GDPs had not heard of the IOTN. Of those who had, the lack of a fee was the main reason for not using it. GDPs also passed on the 'gatekeeper' role for treatment need assessment to OS. OS did not use IOTN because it was not mandatory. These points serve to reinforce the perceived lack of salience of the questionnaire topic and suggest that respondents place little value on IOTN use.

Mandatory use of the IOTN for all orthodontic assessments and an 'IOTN fee' are obvious starting points to increase usage. However, two other strategies seem equally valid in order to promote usage: increasing the IOTN's profile (particularly in the GDP population) and meeting perceived, locally accessed, educational demand. Other incentives have been suggested, such as improving the practitioner working conditions by increasing case variety and reducing ambiguities over who is eligible for NHS treatment. ${ }^{8}$

There were both perceived benefits and drawbacks to potential IOTN introduction. GDPs perceived the main benefit to be the setting of national standards of practice, thereby reducing ambiguity as to who has greater treatment need: IOTN use could reduce the variability of orthodontic assessments. This should shift the allocation of public resources from demand onto need, helping to reconcile "the increasing demands for orthodontic treatment within a framework of finite resources'2 and thereby helping dentists deliver costeffective treatment assessments. ${ }^{1}$ OS were statistically significantly less likely to cite the same reason: they are more consistent as to what they perceive as treatment need when compared to other orthodontic providers. ${ }^{23}$ In the context of this survey, OS appear to have greater self-confidence in needs assessment.

The main drawbacks to mandatory IOTN use are a perceived restriction in access to orthodontic care, coupled with concern that a practitioner's autonomy in making treatment decisions would be reduced. However, using the IOTN with a threshold grade to allocate NHS resources is unlikely to result in patients who have malocclusions being denied care. ${ }^{5}$ Clinicians retain autonomy when assessing the urgency of treatment need and therefore the timing and type of intervention provided. They also retain full autonomy in the private sector.

\section{CONCLUSIONS}

There has been little spontaneous use of the IOTN in the SGDS. Respondents to this survey seem to place little value 
on the use of such an index. Where IOTN has found a role is in inter-colleague communication and for grading case complexity. There appears to be confusion between the index's ability to determine treatment need and its inability to determine the potential complexity of a case. This differs from that reported in secondary and tertiary care, where usage centres on waiting list control and planning orthodontic services, and where the IOTN's inability to assess case complexity was considered a disadvantage. ${ }^{2,7}$

Two methods would reportedly increase IOTN usage: a fee, and mandatory IOTN use when carrying out orthodontic assessments. Remuneration for use would need to be established as cost-effective when allocating finite NHS resources. Mandatory usage may prove contentious and respondents differed over the positive aspects of IOTN use. The reduction in ambiguity when assessing treatment need was most appealing to GDPs, while the ability to justify treatment allocation to the public appealed to the OS.

Before introduction is mooted, the following issues need careful consideration: the profile of the index needs to be raised, the positive perceptions of use must be highlighted, and perceived educational demand should be met on a local basis. Additionally, two main concerns need to be addressed: the perceived reduction in patient access to orthodontic care, and the perceived lack of clinical autonomy.

This paper forms part of the research conducted during the lead author's higher training fellowship. John Ho-A-Yun and Fay Crawford conceived the idea, and designed and conducted the research; Jan Clarkson contributed to the research protocol and the research process. All three contributed to this manuscript. The lead author is the corresponding author and guarantor of this paper.

We are grateful to other contributors: James Newton, who gave his support throughout the higher training course; Peter Donnan and Zoann Nugent assisted with sample size calculation and statistical analysis; Marie Pitkethly and Margaret Feeney, at East ReN, assisted with the pilot studies; Colin Tilley assisted with the GDP list; Louise Cardno provided secretarial support; and Denis Rice contributed to the clarity of the paper.

We would like to thank all the dentists who took the time to respond to the questionnaire.

John Ho-A-Yun was funded by the Scottish Council for Postgraduate Dental and Medical Education via the University of Dundee. Additional funding for the pilots was made available by East ReN. Fay Crawford is funded by the Chief Scientist Office, Scottish Executive.

1. ter Heege G (ed). Euro-Qual. Towards a quality system for European orthodontic professionals. Amsterdam: IOS Press, 1997.

2. de Oliveira $\mathrm{C}$ M. The planning, contracting and monitoring of orthodontic services, and the use of the IOTN index: a survey of consultants in dental public health in the United Kingdom. Br Dent J 2003: 195: 704-706.

3. Brook P H, Shaw W C. The development of an index of orthodontic treatment priority. Eur J Orthod 1989; 11: 309-320.

4. Lunn H, Richmond S, Mitropoulos C. The use of the Index of Orthodontic Treatment Need (IOTN) as a public health tool: a pilot study. Community Dent Health 1993: 10: 111-121.

5. Green J, O'Brien K. The influence of setting 'cut off' points for orthodontic treatment need upon the reliability of the index of orthodontic treatment need. Br J Orthod 1994; 21: 287-289.

6. Willmot D R, DiBiase D, Birnie D J, Heesterman $R$ A. The Consultant Orthodontists Group survey of hospital waiting lists and treated cases. $\mathrm{Br} J$ Orthod 1995; 22: 53-57.
7. Holmes A, Willmot D R. The Consultant Orthodontists Group 1994 survey of the use of the Index of Orthodontic Treatment Need (IOTN). Br J Orthod 1996; 23: 57-59.

8. Hill K, Goodwin N, Morris J, Hall, A, McLeod H, Burke T. Personal dental services (PDS) pilots: final report of the national evaluation. Birmingham: School of Dentistry and Health Services Management Centre, 2001. ISBN 0704424169.

9. NHS Quality Improvement Scotland. Draft national standards for dental services. Edinburgh: Scottish Executive, 2004. ISBN 0755941837.

10. Scottish Executive. Modernising NHS dental services in Scotland. Consultation. Edinburgh: Scottish Executive, 2003. ISBN 0755940296.

11. Shaw W C, Richmond S, O'Brien K D. The use of occlusal indices: a European perspective. Am J Orthod Dentofacial Orthop 1995; 107: 1-10.

12. Waring $D$, Jones J W. Does the GDP need to know about IOTN? Dent Update 2003; 30: 123-130.

13. McColl $E$, Jacoby $A$, Thomas $L$ et al. Design and use of questionnaires: a review of best practice applicable to surveys of health service staff and patients. Health Technol Assess 2001; 5(31): 1-256.

14. Edwards $P$, Roberts I, Clarke M et al. Increasing response rates to postal questionnaires: systematic review. BMJ 2002; 324: 1183-1185.

15. Tan R T, Burke FJ T. Response rates to questionnaires mailed to dentists. A review of 77 publications. Int Dent J 1997; 47: 349-354.

16. Streiner D, Norman L. Health measurement scales. Oxford: Oxford University Press, 1995.

17. Bryman A, Burgess R (eds). Analysing qualitative data. London: Routledge, 1993.

18. Pope C, Mays N (eds). Qualitative research in healthcare. Bristol: BMJ Publishing Group, 2000.

19. Silverman D. Doing qualitative research. London: Sage, 2000.

20. Malterud K. Shared understanding of the qualitative research process. Guidelines for the medical researcher. Fam Pract 1993; 10: 201-206.

21. Heberlein TA, Baumgartner R. Factors affecting response rates to mailed questionnaires: a quantitative analysis of the published literature. Am Sociol Rev 1978; 43: 447-462.

22. Ho-A-Yun J, Crawford F, Newton J, Clarkson J. The effect of advance telephone prompting in a survey of general dental practitioners in Scotland: a randomised controlled trial. Community Dent Health 2007: 24: 233-237.

23. Richmond S, O'Brien K, Roberts $C$, Andrews M. Dentists' variation in the determination of orthodontic treatment need. Br J Orthod 1994; 21: 65-68. 